\title{
Componente epifítico vascular de um fragmento florestal urbano, município de Criciúma, Santa Catarina, Brasil
}

\author{
Lislaine Cardoso de Oliveira \\ Peterson Teodoro Padilha \\ Emilaine Biava Dalmolin \\ Telma Elyta Vilhalba Azeredo \\ Vanilde Citadini-Zanette * \\ Universidade do Extremo Sul Catarinense - UNESC \\ Bairro Universitário, Herbário sala 10, Bloco Biblioteca \\ CEP 88806-000, Criciúma - SC, Brasil \\ *Autor para correspondência \\ vcz@unesc.net
}

Submetido em 25/04/2012

Aceito para publicação em 18/03/2013

\section{Resumo}

Este estudo teve por objetivo realizar levantamento florístico e fitossociológico, bem como analisar a distribuição espacial do componente epifítico vascular em um fragmento florestal em área urbana pertencente à floresta ombrófila densa submontana no município de Criciúma-SC. Adicionalmente, informações sobre os grupos ecológicos das espécies epifíticas e as estratégias de polinização e de dispersão também são apresentadas. Foram amostradas 60 árvores como forófitos com DAP $\geq 10 \mathrm{~cm}$, pelo método de pontos quadrantes centrados, e foi utilizado o método expedito por caminhamento para registrar as espécies epifíticas não amostradas nos forófitos pelo método fitossociológico. A frequência foi avaliada com base na ocorrência dos epífitos nos forófitos e nos segmentos fuste e copa, quando registrada a presença dos epífitos nos dois segmentos. Foram encontradas 65 espécies distribuídas em 39 gêneros e em 14 famílias, das quais 49 foram amostradas no levantamento fitossociológico e as demais no levantamento por caminhamento. Bromeliaceae apresentou a maior riqueza, seguida de Orchidaceae e Cactaceae. Tillandsia recurvata (L.) L. foi citada pela primeira vez para o sul do estado. A diversidade específica foi estimada em $\mathrm{H}^{\prime}=3,33$ e a equabilidade (E) foi igual a 0,86 . $\mathrm{O}$ grupo ecológico dos holoepífitos foi o mais representativo na área de estudo. Entomofilia e anemocoria foram as estratégias predominantes para polinização e dispersão, respectivamente. No levantamento fitossociológico, o número de espécies epifíticas nos forófitos variou de 0 a 21. Os maiores valores de importância foram os relativos à Rhipsalis teres (Vell.) Steud. e Microgramma vacciniifolia (Langsd. \& Fisch.) Copel.

Palavras-chave: Fitossociologia; Floresta ombrófila densa; Florística

\section{Abstract}

Vascular epiphytic component in an urban forest fragment in Criciuma, Santa Catarina, Brazil. This study aimed to conduct a floristic and phytosociological survey, as well as analyze the spatial distribution of the vascular epiphytic component in an urban forest fragment belonging to the submontane dense ombrophilous forest in the town of Criciuma, Santa Catarina, Brazil. In addition, information on the ecological groups of epiphytic 
species and the strategies for pollination and dispersal were also presented. One sampled 60 trees as phorophytes with $\mathrm{DBH} \geq 10 \mathrm{~cm}$, through the point-centered quarter method, and the expeditious walking method was used for recording the epiphytic species which weren't sampled in the phorophytes through the phytosociological method. The frequency was evaluated having the occurrence of epiphytes in the phorophytes and the segments on the bole and crown as a basis. One recorded the presence of epiphytes in the phorophytes in the segments on the bole and crown. One found 65 species distributed into 39 genera and 14 families, out of which 49 were sampled in the phytosociological survey and the remaining ones in the walking survey. Bromeliaceae showed the highest richness, followed by Orchidaceae, and Cactaceae. Tillandsia recurvata (L.) L. was firstly mentioned in the southern state. The specific diversity was estimated as $\mathrm{H}^{\prime}=3.33$ and evenness (E) was equal to 0.86 . The ecological group of holoepiphytes was the most representative one in the area under study. Entomophily and anemochory were the prevailing strategies for pollination and dispersal, respectively. In the phytosociological survey, the number of epiphytic species in the phorophytes ranged from 0 to 21 . The highest importance values were those related to Rhipsalis teres (Vell.) Steud. and Microgramma vacciniifolia (Langsd. \& Fisch.) Copel.

Key words: Dense ombrophilous forest; Floristic; Phytosociology

\section{Introdução}

De todas as espécies vasculares existentes, $10 \%$ são epífitos (KRESS, 1986), que somam aproximadamente 29.000 espécies, pertencentes a 876 gêneros e a 84 famílias (GENTRY; DODSON, 1987). Por definição, plantas epífitas são nutricionalmente independentes de seus hospedeiros, utilizando-os somente como suporte para fixação (COXSON; NADKARNI, 1995). Esta interação entre epífitas e sua árvore hospedeira é conhecida como comensalismo, onde o forófito não é afetado negativamente enquanto provê suporte físico para as epífitas que se beneficiam com condições favoráveis ao seu crescimento (BENZING, 1995).

Os epífitos têm um importante papel na conservação da biodiversidade de florestas tropicais (HOELTGEBAUM, 2003), sendo expressivos elementos na composição desta flora (GENTRY; DODSON, 1987). Este grupo de plantas proporciona recursos alimentares como frutos, néctar, pólen e água, além de microambientes especializados para a fauna constituída por uma infinidade de organismos voadores, arborícolas e escansoriais (COIMBRA FILHO; ALDRIGHI, 1972). A biomassa epifítica também exerce grande influência sobre a ciclagem de água e nutrientes no interior da floresta (INGRAM; NADKARNI, 1993).

O padrão de distribuição espacial das espécies de epífitos vasculares varia basicamente nos sentidos horizontal, dado pelas sua ocorrência em diferentes regiões geográficas, tipos florestais e forófitos e, vertical, quando sua distribuição varia ao longo da extensão da árvore (REITZ, 1983; TER STEEGE; CORNELISSEN, 1989). Esse padrão de distribuição é determinado por fatores como: luminosidade e umidade (TER STEEGE; CORNELISSEN, 1989; BENZING, 1995), arquitetura e interceptação diferencial da chuva pelas copas das árvores (BENZING, 1990; HERWITZ; SLYE, 1992), dimensões dos forófitos (CATLING; LEFKOVITCH, 1989), textura e estabilidade do ritidoma (TER STEEGE; CORNELISSEN, 1989), reprodução dos indivíduos, movimento e fixação das sementes ao substrato, germinação (GARCIA-FRANCO; RICO-GRAY, 1988), crescimento e sobrevivência das plântulas (HIETZ, 1997; RUDOLPH et al., 1998).

No Brasil, estudos com o componente epifítico vascular são recentes, sendo realizados principalmente nas regiões sul e sudeste, como os de Waechter (1986; 1992; 1998); Dislich e Mantovani (1998); Breier (1999; 2005); Kersten e Silva (2001; 2002); Rogalski e Zanin (2003); Bonnet e Queiroz (2006); Azeredo (2010); Kersten e Waechter (2011), entre outros, muito embora em alguns deles o estudo tenha enfocado somente famílias botânicas específicas (CAGLIONI et al., 2012).

Tendo em vista o aumento acelerado da fragmentação da Mata Atlântica e a relevância dos epífitos vasculares para a estrutura e dinâmica desse bioma, este estudo objetivou realizar levantamento florístico e fitossociológico, bem como analisar a distribuição espacial do componente epifítico vascular de um fragmento florestal em área urbana, no sul do estado 
de Santa Catarina. Registraram-se também os grupos ecológicos e as estratégias de polinização e dispersão das espécies epifíticas.

\section{Material e Métodos}

\section{Área estudada}

A área de estudo pertence ao Parque Ecológico Municipal José Milanese (PEMJM), criado de acordo com a lei Municipal n 2.856/1993 (CMC, 2006) a fim de preservar espécies nativas da fauna e flora da Mata Atlântica regional. O PEMJM situa-se no município de Criciúma, sul do estado de Santa Catarina (28 48'S e $49^{\circ} 25^{\prime} \mathrm{W}$, altitude $34 \mathrm{~m}$ ) (Figura 1) e ocupa área total de 7,7 ha, sendo 5,2 destes, cobertos por Floresta Ombrófila Densa Submontana. No local foram registradas 137 espécies arbóreas, pertencentes a 45 famílias com o predomínio de Myrtaceae (30), Lauraceae (14) e Rubiaceae (7), com indivíduos de grande porte que atingem mais de $20 \mathrm{~m}$ de altura (SILVA, 2006).
A vegetação herbácea terrícola do interior do Parque está representada principalmente por Coccocypselum condalia Pers. e Ichnanthus pallens (Sw.) Munro ex Benth. (ROCHA, 2007). Como espécies com potencialidades medicinais, Brum-Figueiró, A. C.; Citadini-Zanette (2008) citam em seu trabalho Casearia sylvestris Swartz (Chá-de-bugre), Protium kleinii Cuatrec (Almesca), Garcinia gardneriana (Planch. et Triana) Zappi (Bacupari), entre outras.

Segundo o sistema de classificação de KöppenGeiger (KOTTEK et al., 2006), o clima da região enquadra-se no tipo Cfa (subtropical úmido com verões quentes). A temperatura média anual varia de 17,0 a $19,3^{\circ} \mathrm{C}$, sendo a média das máximas entre 23,4 e $25,9^{\circ} \mathrm{C}$, e das mínimas de 12,0 e $15,1^{\circ} \mathrm{C}$. A umidade relativa do ar é, em média, $81,8 \%$. A precipitação média anual na região carbonífera catarinense é de 1.400 a $1.600 \mathrm{~mm} /$ ano, sem índices pluviométricos mensais inferiores a 60 mm (EPAGRI, 2001).

FIGURA 1: Vista aérea do Parque Ecológico Municipal José Milanese (2848'S e 49²5'W, altitude 34 m), Criciúma, Santa Catarina, Brasil. Fonte: Google Earth em 10 de abril de 2012, adaptado.

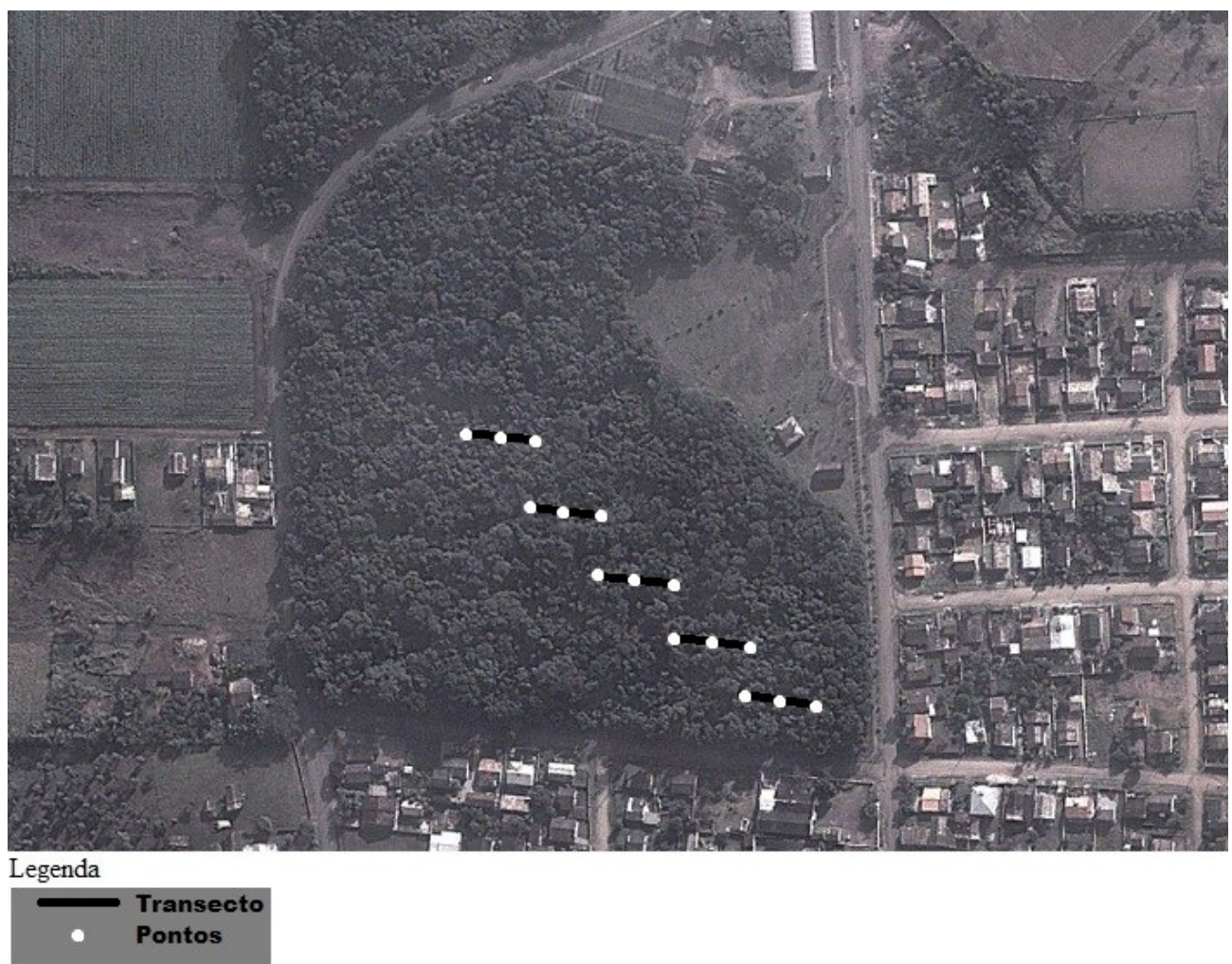




\section{Amostragem}

As incursões à área ocorreram mensalmente pelo período de 12 meses entre os anos de 2009 e 2010 . O levantamento fitossociológico foi realizado utilizandose o método de quadrantes centrados (COTTAM; CURTIS, 1956), considerando cada árvore como uma unidade amostral. Foram estabelecidos, no fragmento, cinco transecções, separadas $20 \mathrm{~m}$ entre si. Para cada transecção foram determinados três pontos quadrantes, separados entre si por $10 \mathrm{~m}$, totalizando 60 árvores amostradas. Registrou-se a presença de epífitas em forófitos com diâmetros à altura do peito (DAP) maior ou igual a $10 \mathrm{~cm}$. No estudo da distribuição vertical, os forófitos foram divididos em fuste e copa. Para caracterização da distribuição horizontal dos epífitos calcularam-se as frequências relativas percentuais nos forófitos e nos segmentos dos forófitos (GIONGO; WAECHTER, 2004). Para as espécies não incluídas na amostragem fitossociológica, utilizou-se o método expedito por caminhamento (FILGUEIRAS et al., 1994).

Utilizou-se o teste $\chi^{2}$, ao nível de significância de 5\% (ZAR, 1999; CALLEGARI-JACQUES, 2003), para determinar diferenças entre frequência observada e esperada das epífitas nas duas zonas verticais propostas. Os valores esperados de cada espécie epifítica foram obtidos dividindo-se por dois a frequência observada sobre indivíduos forofíticos. As espécies que obtiveram frequências esperadas inferiores a cinco não foram consideradas. Foi aplicada a correção de Yates $(-0,5)$ ao teste $\chi^{2}$.

A relação entre o número de espécies epífitas e o diâmetro do forófito foi testada através de análise de correlação de Spearman (ZAR, 1999), ao nível de significância a 5\%. A análise foi feita com o auxílio do software PAST, versão 1.89 (HAMMER et al., 2009).

Para análise da heterogeneidade florística da área estudada foram utilizados os índices de Shannon (H') para obtenção da diversidade específica (alfa) e de equabilidade (E) de acordo com MAGURRAN (1988) e PIELOU (1975), respectivamente.

Adicionalmente, como dados secundários, as espécies foram classificadas em categorias ecológicas, conforme Benzing (1990) e quanto à biologia reprodutiva (polinização e dispersão) estabelecidas com base na literatura (FISCHER; ARAÚJO, 1995; SANTOS, 2000; BORGO; SILVA, 2003; HEFLER; FAUSTIONI, 2004; BREIER, 2005; DETTKE et al., 2008; SANTOS, 2008).

O material fértil foi coletado, herborizado e incorporado ao Herbário Pe. Dr. Raulino Reitz (CRI) da Universidade do Extremo Sul Catarinense - UNESC, Criciúma, Santa Catarina.

\section{Resultados}

Foram registradas na área de estudo, 65 espécies epifíticas, distribuídas em 39 gêneros e em 14 famílias (Tabela 1). Destas espécies, 49 foram amostradas no levantamento fitossociológico e as demais no levantamento florístico. A família com maior riqueza específica foi Bromeliaceae (19), sendo Tillandsia recurvata (L.) L., citada pela primeira vez para o sul do estado de Santa Catarina, seguida de Orchidaceae (14) e Cactaceae (7). As demais famílias apresentaram número igual ou inferior a quatro espécies. O gênero com maior diversidade foi Tillandsia com seis espécies, seguido de Vriesea e Rhipsalis, com cinco espécies e Aechmea e Peperomia com quatro espécies. Para os demais gêneros foram registrados número igual ou inferior a duas espécies. As espécies com maior ocorrência nos forófitos, foram Rhipsalis teres (Vell.) Steud. (35 forófitos), Microgramma vacciniifolia (Langsd. \& Fisch.) Copel. (23), Vriesea gigantea (Mart. ex Schult. f.) Mez (22) e Microgramma squamulosa (Kaulf.) de la Sota (21). As demais espécies foram registradas em quantidade igual ou inferior a 15 forófitos (Tabela 2).

Para as categorias ecológicas, os holoepífitos contribuíram com 59 espécies $(90,77 \%)$, sendo 55 holoepífitos verdadeiros, dois holoepífitos facultativos e dois holoepífitos acidentais; os hemiepífitos representaram seis das espécies registradas (9,23\%), com três hemiepífitos primários e três hemiepífitos secundários (Tabela 1). 
TABELA 1: Espécies epifíticas vasculares do Parque Ecológico Municipal José Milanese, Criciúma, Santa Catarina, Brasil, onde: Categoria Ecológica (CE): hololoepífitas verdadeiras (HLV), holoepífita facultativa (HLF), holoepífita acidental (HLA) hemiepífita primária (HMP), hemiepífita secundária (HMS); Estratégias de polinização (EP) e dispersão de diásporos (ED): entomofilia (EN), quiropterofilia (QF), ornitofilia (OR) e anemofilia (AF); zoocoria (ZO), quiropterocoria (QU) anemocoria (AN) pogonocoria (PO), autocoria (AU), epizoocoria (EP) e sem identificação (SI).

\begin{tabular}{|c|c|c|c|}
\hline FAMÍLIA/Espécie & $\mathbf{C E}$ & EP & ED \\
\hline \multicolumn{4}{|l|}{ ARACEAE } \\
\hline Anthurium gaudichaudianum Kunth & HLV & EN & $\mathrm{ZO}$ \\
\hline Anthurium scandens (Aubl.) Engl. & HLV & EN & $\mathrm{ZO}$ \\
\hline Philodendron appendiculatum Nadruz \& Mayo & HMP & EN & $\mathrm{ZO}$ \\
\hline Philodendron bipinnatifidum Schott ex Endl. & HMP & EN & $\mathrm{ZO}$ \\
\hline \multicolumn{4}{|l|}{ ASPLENIACEAE } \\
\hline Asplenium scandicinum Kaulf. & HLV & - & AN \\
\hline \multicolumn{4}{|l|}{ BEGONIACEAE } \\
\hline Begonia radicans Vell. & HMS & EN & AN \\
\hline \multicolumn{4}{|l|}{ BLECHNACEAE } \\
\hline Blechnum binervatum (Poir.) C.V. Morton \& Lellinger & HMP & - & AN \\
\hline \multicolumn{4}{|l|}{ BROMELIACEAE } \\
\hline Aechmea caudata Lindm. & HLV & OR & $\mathrm{ZO}$ \\
\hline Aechmea gamosepala Wittm. & HLV & OR - EN & $\mathrm{ZO}$ \\
\hline Aechmea nudicaulis (L.) Griseb. & HLV & OR & $\mathrm{ZO}$ \\
\hline Aechmea recurvata (Klotzsch) L.B. Sm. & HLV & EN & $\mathrm{PO}$ \\
\hline Billbergia nutans H. Wendl. ex Regel & HLV & OR & $\mathrm{PO}$ \\
\hline Billbergia zebrina (Herb.) Lindl. & HLV & OR & QU \\
\hline Canistrum fragrans (Linden) Mabb. & HLF & OR & $\mathrm{ZO}$ \\
\hline Canistrum superbum (Lindm.) Mez & HLV & OR & $\mathrm{ZO}$ \\
\hline Tillandsia geminiflora Brongn. & HLV & OR - EN & PO \\
\hline Tillandsia mallemontii Glaz. ex Mez & HLV & EN & $\mathrm{PO}$ \\
\hline Tillandsia recurvata $(\mathrm{L}.) \mathrm{L}$. & HLV & $\mathrm{EN}$ & $\mathrm{PO}$ \\
\hline Tillandsia stricta Sol. ex Sims & HLV & OR - EN & $\mathrm{PO}$ \\
\hline Tillandsia tenuifolia $\mathrm{L}$. & HLV & EN & $\mathrm{PO}$ \\
\hline Tillandsia usneoides (L.) L. & HLV & EN & $\mathrm{PO}$ \\
\hline Vriesea carinata Wawra & HLV & OR & $\mathrm{PO}$ \\
\hline Vriesea flammea L.B. Sm. & HLV & OR & $\mathrm{PO}$ \\
\hline Vriesea gigantea Mart. ex Schult. f. & HLV & $\mathrm{OR}-\mathrm{QF}$ & $\mathrm{PO}$ \\
\hline Vriesea incurvata Gaudich. & HLV & OR & $\mathrm{PO}$ \\
\hline Vriesea vagans (L.B. Sm.) L.B. Sm. & HLV & OR & $\mathrm{PO}$ \\
\hline \multicolumn{4}{|l|}{ CACTACEAE } \\
\hline Lepismium cruciforme (Vell.) Miq. & HLV & EN & $\mathrm{ZO}$ \\
\hline Lepismium sp. & HLV & EN & $\mathrm{ZO}$ \\
\hline Rhipsalis pachyptera Pfeiff. & HLV & EN & $\mathrm{ZO}$ \\
\hline Rhipsalis paradoxa (Salm-Dyck ex Pfeiff.) Salm-Dyck & HLV & EN & $\mathrm{ZO}$ \\
\hline Rhipsalis sp. & HLV & EN & $\mathrm{ZO}$ \\
\hline
\end{tabular}


Rhipsalis teres (Vell.) Steud.

$\begin{array}{lll}\text { HLV } & \text { EN } & \text { ZO } \\ \text { HLV } & \text { EN } & \text { ZO } \\ & & \\ \text { HMS } & - & \text { AN } \\ \text { HMS } & - & \text { AN } \\ \text { HLF } & - & \text { AN }\end{array}$

Rhipsalis trigona Pfeiff.

Lomagramma guianensis (Aubl.) Ching

AN

Rumohra adiantiformis (G. Forst.) Ching

HLF

GESNERIACEAE

Codonanthe devosiana Lem.

HLV

EN

HLV

OR

AU

Nematanthus fissus (Vell.) L.E. Skog

HLA

EN

$\mathrm{ZO}$

Leandra dasytricha (A. Gray) Cogn.

HLV

HLV

EN

AN

Acianthera sp.

HLV

EN

AN

Brassavola tuberculata Hook.

HLV

EN

AN

Epidendrum sp.

EN

AN

Isabelia pulchella (Kraenzl.) Senghas \& Teusch.

HLV

EN

AN

Maxillaria marginata Fenzl

HLV

EN

AN

Octomeria crassifolia Lindl.

HLV

EN

AN

Oncidium cf. flexuosum (Kunth) Lindl.

HLV

EN

AN

Ornithocephalus myrticola Lindl.

HLV

EN

AN

Pleurothallis grobyi Bateman ex Lindl.

HLV

EN

AN

Polystachya concreta (Jacq.) Garay \& Sweet

HLV

EN

AN

Prosthechea vespa (Vell.) W.E. Higgins

HLV

EN

AN

HLV

EN

AN

Trichocentrum pumilum (Lindl.) M.W. Chase \& N.H. Williams

HLV

EN

AN

PIPERACEAE

Peperomia catharinae Miq.

HLV

AF

EP

Peperomia glabella (Sw.) A. Dietr.

HLV

HLV

$\mathrm{AF}$

EP

Peperomia myrtifolia (Vahl) A. Dietr.

HLV

AF

EP

Peperomia pereskiifolia (Jacq.) HBK.

AF

EP

POLYPODIACEAE

Campyloneurum nitidum (Kaulf.) C. Presl.

HLV

HLV

Microgramma squamulosa (Kaulf.) de la Sota

HLV

Microgramma vacciniifolia (Langsd. \& Fisch.) Copel.

HLV

Pleopeltis hirsutissima (Raddi) de la Sota

HLV

Pleopeltis pleopeltifolia (Raddi) Alston

HLV

AN

Serpocaulon catharinae (Langsd. \& Fisch.) A.R. Sm.

HLV

AN

AN

AN

AN

PTERIDACEAE

Vittaria lineata (L.) $\mathrm{Sm}$.

HLA

$-$

AN

SOLANACEAE

Solandra grandiflora Salisb.

HLA

OR

SI 
TABELA 2: Espécies epifíticas vasculares amostradas no levantamento fitossociológico no Parque Ecológico Municipal José Milanese, Criciúma, Santa Catarina, Brasil, em ordem decrescente de valor de importância. Npi = número de forófitos ocupados pela espécie epifítica i; Nfi = número de fustes ocupados pelas espécies epifíticas i; Nci = número de copas ocupadas pelas espécies epifíticas i; FRpi = frequência relativa da espécie i nos forófitos; FRci $=$ frequência relativa da espécie i nas copas; FRfi = frequência relativa da espécie i nos fustes; VIe = valor de importância da espécie epifítica i.

\begin{tabular}{|c|c|c|c|c|c|c|c|}
\hline Espécie & Npi & Nfi & Nci & FRpi & FRfi & FRci & VIe \\
\hline Rhipsalis teres & 35 & 13 & 25 & 11,15 & 10,74 & 10,92 & 10,83 \\
\hline Microgramma vacciniifolia & 25 & 10 & 23 & 7,96 & 8,25 & 10,04 & 9,15 \\
\hline Vriesea gigantea & 22 & 4 & 20 & 7,01 & 3,31 & 8,73 & 6,02 \\
\hline Microgramma squamulosa & 21 & 4 & 19 & 6,68 & 3,31 & 8,30 & 5,80 \\
\hline Anthurium gaudichaudianum & 15 & 7 & 13 & 4,77 & 5,78 & 5,67 & 5,73 \\
\hline Tillandsia stricta & 18 & 5 & 13 & 5,72 & 4,12 & 5,67 & 4,90 \\
\hline Rhipsalis sp. & 18 & 5 & 13 & 5,72 & 4,12 & 5,67 & 4,90 \\
\hline Rhipsalis trigona & 15 & 6 & 10 & 4,77 & 4,95 & 4,37 & 4,66 \\
\hline Vittaria lineata & 11 & 11 & 0 & 3,50 & 9,08 & 0,00 & 4,55 \\
\hline Pleopeltis pleopeltifolia & 16 & 3 & 13 & 5,10 & 2,48 & 5,67 & 4,08 \\
\hline Rhipsalis paradoxa & 12 & 5 & 8 & 3,82 & 4,13 & 3,49 & 3,81 \\
\hline Pleopeltis hirsutissima & 8 & 4 & 7 & 2,55 & 3,31 & 3,06 & 3,18 \\
\hline Aechmea nudicaulis & 10 & 1 & 9 & 3,18 & 0,83 & 3,93 & 2,38 \\
\hline Canistrum superbum & 7 & 2 & 7 & 2,23 & 1,65 & 3,06 & 2,35 \\
\hline Oncidium cf. flexuosum & 7 & 4 & 3 & 2,23 & 3,31 & 1,31 & 2,31 \\
\hline Anthurium scandens & 5 & 3 & 3 & 1,59 & 2,48 & 1,31 & 1,89 \\
\hline Lepismium cruciforme & 3 & 3 & 3 & 0,96 & 2,48 & 1,31 & 1,89 \\
\hline Lomagramma guianensis & 4 & 3 & 2 & 1,27 & 2,48 & 0,87 & 1,68 \\
\hline Blechnum binervatum & 4 & 4 & 0 & 1,27 & 3,31 & 0,00 & 1,65 \\
\hline Philodendron appendiculatum & 4 & 1 & 4 & 1,27 & 0,83 & 1,75 & 1,29 \\
\hline Tillandsia mallemontii & 4 & 1 & 4 & 1,27 & 0,83 & 1,75 & 1,29 \\
\hline Peperomia catharinae & 3 & 3 & 0 & 0,96 & 2,48 & 0,00 & 1,24 \\
\hline Codonanthe sp. & 5 & 0 & 5 & 1,59 & 0,00 & 2,18 & 1,09 \\
\hline Tillandsia usneoides & 3 & 1 & 3 & 0,96 & 0,83 & 1,31 & 1,07 \\
\hline Canistrum fragans & 4 & 0 & 4 & 1,27 & 0,00 & 1,75 & 0,87 \\
\hline Tillandsia tenuifolia & 3 & 1 & 2 & 0,96 & 0,83 & 0,87 & 0,85 \\
\hline Codonanthe devosiana & 2 & 2 & 0 & 0,64 & 1,65 & 0,00 & 0,83 \\
\hline Peperomia pereskiifolia & 2 & 2 & 0 & 0,64 & 1,65 & 0,00 & 0,83 \\
\hline Peperomia sp. & 2 & 2 & 0 & 0,64 & 1,65 & 0,00 & 0,83 \\
\hline Aechmea recurvata & 3 & 0 & 3 & 0,96 & 0,00 & 1,31 & 0,66 \\
\hline Billbergia nutans & 2 & 1 & 1 & 0,64 & 0,83 & 0,44 & 0,63 \\
\hline Tillandsia geminiflora & 1 & 1 & 1 & 0,32 & 0,83 & 0,44 & 0,63 \\
\hline Lepismium sp. & 2 & 1 & 1 & 0,64 & 0,83 & 0,44 & 0,63 \\
\hline Billbergia zebrina & 2 & 0 & 2 & 0,64 & 0,00 & 0,87 & 0,45 \\
\hline Polystachya concreta & 2 & 0 & 2 & 0,64 & 0,00 & 0,87 & 0,45 \\
\hline
\end{tabular}




\begin{tabular}{|c|c|c|c|c|c|c|c|}
\hline Rhipsalis pachyptera & 1 & 1 & 0 & 0,32 & 0,83 & 0,00 & 0,41 \\
\hline Leandra dasytricha & 1 & 1 & 0 & 0,32 & 0,83 & 0,00 & 0,41 \\
\hline Acianthera sp. & 1 & 1 & 0 & 0,32 & 0,83 & 0,00 & 0,41 \\
\hline Brassavola tuberculata & 1 & 1 & 0 & 0,32 & 0,83 & 0,00 & 0,41 \\
\hline Bifrenaria sp. & 1 & 1 & 0 & 0,32 & 0,83 & 0,00 & 0,41 \\
\hline Epidendrum sp. & 1 & 1 & 0 & 0,32 & 0,83 & 0,00 & 0,41 \\
\hline Octomeria crassifolia & 1 & 1 & 0 & 0,32 & 0,83 & 0,00 & 0,41 \\
\hline Trichocentrum pumilum & 1 & 1 & 0 & 0,32 & 0,83 & 0,00 & 0,41 \\
\hline Vriesea flammea & 1 & 0 & 1 & 0,32 & 0,00 & 0,44 & 0,22 \\
\hline Vriesea vagans & 1 & 0 & 1 & 0,32 & 0,00 & 0,44 & 0,22 \\
\hline Rumohra adiantiformis & 1 & 0 & 1 & 0,32 & 0,00 & 0,44 & 0,22 \\
\hline Maxilaria marginata & 1 & 0 & 1 & 0,32 & 0,00 & 0,44 & 0,22 \\
\hline Ornithocephalus myrticola & 1 & 0 & 1 & 0,32 & 0,00 & 0,44 & 0,22 \\
\hline \multirow[t]{2}{*}{ Solandra grandiflora } & 1 & 0 & 1 & 0,32 & 0,00 & 0,44 & 0,22 \\
\hline & 314 & 121 & 229 & 100,00 & 100,00 & 100,00 & 100,00 \\
\hline
\end{tabular}

Nas estratégias de polinização, 33 espécies (62,26\%) de sete famílias (Araceae, Begoniaceae, Bromeliaceae, Cactaceae, Gesneriaceae, Melastomataceae e Orchidaceae) foram classificadas como entomófilas, 12 espécies (22,64\%) de duas famílias (Bromeliaceae e Gesneriaceae) como ornitófilas, quatro espécies $(7,55 \%)$ de Piperaceae como anemófilas, três espécies (5,66\%) de Bromeliaceae como ornitófilas e entomófilas, uma espécie $(1,89 \%)$ da mesma família como quiropterófila e ornitófila e 12 espécies, por pertencerem ao grupo das samambaias, entram numa categoria não classificável quanto à polinização (Tabela 1). Quanto à dispersão dos diásporos, 27 espécies (41,54\%) de seis famílias (Begoniaceae, Blechnaceae, Bromeliaceae, Orchidaceae, Pteridaceae e Polypodiaceae) são anemocóricas, 17 espécies $(26,15 \%)$ de cinco famílias (Araceae, Bromeliaceae, Cactaceae, Melastomataceae, Piperaceae) são zoocóricas, 13 espécies (20,00\%) de Bromeliaceae são pogonocóricas, quatro espécies $(6,15 \%)$ de Piperaceae são epizoocóricas, duas espécies $(3,08 \%)$ de Gesneriaceae são autocóricas, uma espécie $(1,54 \%)$ de Bromeliaceae como quiropterocórica e para uma espécie $(1,54 \%)$ não foi registrada a estratégia de dispersão (Tabela 1).

Com relação à distribuição horizontal, considerando-se a frequência sobre os indivíduos forofíticos, as espécies de maior valor epifítico (VIe) foram: Rhipsalis teres, Microgramma vacciniifolia, Vriesea gigantea, Microgramma squamulosa e Anthurium gaudichaudianum, respectivamente; as demais espécies apresentaram frequências nos forófitos inferior a 5 (Tabela 2). Na distribuição vertical, as copas apresentaram-se mais colonizadas em relação aos fustes. Rhipsalis teres e Microgramma vacciniifolia foram mais frequentes em ambos os segmentos. O padrão de distribuição vertical das espécies foi de 229 indivíduos na copa e 121 no fuste (Tabela 2).

O teste $\chi^{2}$ revelou que seis espécies (Microgramma vacciniifolia, Vriesea gigantes, Microgramma squamulosa, Vittaria lineata, Pleopeltis pleopeltifolia e Aechmea nudicalis), apresentaram diferenças estatisticamente significativas em frequência das epifítas por local de fixação $(p<0,05)$. Das seis espécies, apenas Vittaria lineata teve preferência pelo fuste, as demais pelo tronco.

Os valores obtidos por meio da análise de correlação de Spearman mostraram forte correlação positiva entre o DAP e riqueza de epífitas ( $\mathrm{rs}=0,5936$; $\mathrm{p}<0,001$ ), evidenciando que árvores com diâmetros maiores apresentam maior riqueza de espécies, por oferecer maior área como substrato (BENZING, 1990; BONNET; QUEIROZ, 2006). 
O índice de Shannon (H') estimado para a diversidade específica foi de 3,33, e a equabilidade (E) igual a 0,86 . A riqueza epifítica variou de zero a 21 nos forófitos amostrados. O maior valor (21) foi verificado em um indivíduo de Ocotea indecora (Lauraceae) com $50 \mathrm{~cm}$ de DAP e $12 \mathrm{~m}$ de altura total. Ainda se destacaram, Meliosma sellowii (19) com $38 \mathrm{~cm}$ de DAP e $15 \mathrm{~m}$ de altura e Ocotea puberula (16) com $60 \mathrm{~cm}$ de DAP e $14 \mathrm{~m}$ de altura. Não foram encontrados epífitos em 12 indivíduos arbóreos.

\section{Discussão}

Considerando que se trata de um fragmento localizado no meio urbano com área reduzida, o número de espécies de epífitos vasculares registrado está próximo ao encontrado em outras áreas no sul do país (WAECHTER, 1992; 1998; BREIER, 1999; DITTRICH et al., 1999; KERSTEN; SILVA, 2001; GERALDINO et al., 2010), que apresentam um padrão de riqueza específica elevado em relação ao número de famílias, conforme apresentado por Dias (2009).

Bromeliaceae e Orchidaceae que destacaram-se no presente estudo, estão entre as famílias mundialmente mais ricas da flora epifítica (MADISON, 1977; KRESS, 1986; BENZING, 1990). Bromeliaceae, a mais abundante, também foi predominante no estudo de Dettke et al. (2008), embora, via de regra, Orchidaceae é a que destaca-se em riqueza nos estudos com epífitos no Brasil (ROGALSKI; ZANIN, 2003; BORGO; SILVA, 2003; GIONGO; WAECHTER, 2004; KERSTEN; WAECHTER, 2011), independente de pluviosidade (DISLICH; MANTOVANI, 1998). Por tratar-se de plantas ornamentais, com significativo valor econômico, as orquídeas são alvos constantes de coleta predatória (VENTURA et al., 2002) e tratando-se de fragmento urbano, o acesso a estas plantas é mais facilitado, podendo ser a causa do menor número de Orchidaceae na área, um dos fatores também atribuído por Dettke et al. (2008) em seu estudo na Floresta Estacional Semidecidual.

Tillandsia recurvata (L.) L. foi citada pela primeira vez para o sul de Santa Catarina, mesmo que encontrada em outras localidades do Estado, o que demonstra a falta de estudos na região, muito embora Reitz (1983) tenha realizado um intenso e amplo levantamento de Bromeliaceae no estado de Santa Catarina.

A maior representatividade de holoepífitos, como ocorre neste estudo, é generalizada tanto no Brasil, como em quase todo o mundo (NIEDER et al., 2001; GIONGO; WAECHTER, 2004; KERSTEN; KUNIYOSHI, 2009; BONNET et al., 2011). A proporção de hemiepífitos é variável, conforme a tipologia florestal estudada (KERSTEN, 2006), apresentando neste trabalho um padrão em conformidade com o de outros estudos em Floresta Ombrófila Densa (FONTOURA et al., 1997; KERSTEN; SILVA, 2002). O baixo número de epífitas facultativas e acidentais ( $6 \%$ do total) também foi encontrado em estudos realizados por Kersten e Kuniyoshi (2009) e Kersten e Waechter (2011) para o Estado do Paraná.

Quanto à estratégia de polinização, houve prevalência de espécies com vetores bióticos, principalmente por insetos. A forma de dispersão mais comum foi anemocoria, favorecida pelo fato de os diásporos anemocóricos apresentarem pequeno tamanho, o que possibilita maior eficiência na colonização por percorrerem longas distâncias até encontrar um substrato propício ao seu desenvolvimento (NIEDER et al., 1996), podendo estabelecer-se sobre pequenas fissuras na casca dos forófitos. O maior número de espécies que possuem sua dispersão pelo ar pode ser explicado pelo fato de que seus diásporos podem vir de áreas vizinhas, propiciando maior riqueza de espécies anemocóricas (BENZING; OTT, 1981). As estruturas que facilitam a flutuação no ar apresentadas por algumas bromeliáceas, também tiveram relevância nesse estudo (Tabela 1).

$\mathrm{Na}$ distribuição horizontal, o valor de importância epifítico (VIe), dado pela soma das frequências relativas de copas e fustes, Rhipsalis teres e Microgramma vacciniifolia foram as espécies com elevada frequência em ambos os segmentos. Rhipsalis teres, espécie pioneira, embora não esteja tão comumente representada com alto valor de importância nos trabalhos com epífitos, segundo Waechter (1992), pertence a um gênero de forte participação na fisionomia da flora epifítica da região sul do Brasil. Adicionalmente Boeni e Pizo (2007), demonstraram que a espécie não tem preferência por tipo de forófito, o que pode 
indicar maior facilidade de estabelecimento. Microgramma vacciniifolia está constantemente entre as espécies de maior valor de importância, possui rizoma reptante que se estende sobre os fustes e ramos, permitindo que a planta ocupe extensas áreas das árvores (DISLICH; MONTOVANI, 1998; WAECHTER, 1998; KERSTEN; SILVA, 2001; GONÇALVES; WAECHTER, 2003).

A maior colonização de copas em relação aos fustes, na distribuição vertical, salientada em diversos trabalhos, está relacionada à maior área de substrato, diferentes condições de luminosidade e acúmulo de húmus (JOHANSSON, 1974; WAECHTER, 1992; FREIBERG, 1996; RUDOLPH et al., 1998; KERSTEN; SILVA, 2002). Estas condições ambientais possibilitam o estabelecimento de epífitos com diferentes exigências, podendo ser a causa de algumas espécies serem encontradas exclusivamente neste segmento (GIONGO; WAECHTER, 2004). A preferência de Vittaria lineata pelo fuste se dá possivelmente em função de que, em alguns casos, é encontrada também como rupícola (MUSSKOPF, 2006), o que sugere sua preferência por regiões mais baixas no forófito.

Dias (2009), como no presente estudo, obteve resultados positivos em Floresta Ombrófila Densa Submontana quando comparou a riqueza específica de epífitos com o DAP do forófito. Yeaton e Gladstone (1982), Hietz e Hietz-Seifert (1995) e Shaw (2004) acrescentam que forófitos maiores oferecem maior área para fixação das plântulas, maior possibilidade de formação de microclimas diversos, como também pelo maior período de tempo crescendo na floresta, resultando numa forte relação dos grandes indivíduos arbóreos com riqueza de epífitas.

A diversidade indicada pelo índice de Shannon $\left(H^{\prime}=3,33\right)$ foi inferior à encontrada por Petean (2009) na mesma formação florestal no estado do Paraná $\left(\mathrm{H}^{\prime}=4,54\right)$, porém um valor ainda considerado alto quando comparado com de outras formações florestais no sul e sudeste do Brasil (DIAS, 2009), refletindo a importância do fragmento florestal estudado na detenção da biodiversidade epifítica. $\mathrm{O}$ valor do índice de equabilidade de Pielou $(0,86)$ também foi elevado indicando alta uniformidade na distribuição das espécies.
O estudo registrou significativa riqueza específica, que pode estar ameaçada pelas constantes perturbações antrópicas, comuns em áreas urbanas. Trabalhos que agreguem conhecimento quanto à diversidade em fragmentos de florestas são fundamentais para impulsionar a conservação. Tratando-se de epífitas vasculares, indicadoras do grau de distúrbio das florestas (BARTHLOTT et al., 2001), a condição não pode ser diferente, considerando as constantes perturbações florestais e o impacto nas comunidades vegetais, que interferem nos processos ecológicos.

\section{Agradecimentos}

A Universidade do Extremo Sul Catarinense (UNESC) pelo suporte financeiro representado pelas bolsas de Iniciação Científica (PIBIC/UNESC) dos dois primeiros autores e ao Prof. Dr. Jorge Luiz Waechter da Universidade Federal do Rio Grande do Sul pela contribuição na identificação e/ou confirmação de algumas entidades taxonômicas.

\section{Referências}

AZEREDO, T. E. V. Diversidade e distribuição de bromélias epifíticas ao longo de um gradiente altitudinal na Floresta Atlântica do Sul do Brasil. 2010. 54 f. Dissertação (Mestrado em Ciências Ambientais) - Universidade do Extremo Sul Catarinense, Criciúma. 2010.

BARTHLOTT, W.; SCHMIT-NEUERBURG, V.; NIEDER, J.; ENGWALD, S. Diversity and abundance of vascular epiphytes: a comparison of secondary vegetation and primary montane rain forest in the Venezuelan Andes. Plant Ecology, Dordrecht, v. 152, n. 2, p. 145-156, 2001.

BENZING, D. H. Vascular epiphytes. New York: Cambridge University Press, 1990. 354 p.

BENZING, D. H. The physical mosaic and plant variety in forest canopies. Selbyana, Sarasota, v. 16, p. 159-168, 1995.

BENZING, D. H.; OTT, D. W. Vegetative reduction in epiphytic Bromeliaceae and Orchidaceae: its origin and significance. Biotropica, Washington, v. 13, n. 2, p. 131-140, 1981.

BOENI, B. O.; PIZO, M. A. Distribuição vertical, fisiologia e frugivoria de Rhipsalis teres (Cactaceae). In: CONGRESSO DE ECOLOGIA DO BRASIL, 8, 2007, Caxambu. Anais... Caxambu: SBE, 2007. Versão Eletrônica.

BONNET, A.; QUEIROZ, M. H. Estratificação vertical de bromélias epifíticas em diferentes estádios sucessionais da Floresta Ombrófila Densa, Ilha de Santa Catarina, Brasil. Revista Brasileira de Botânica, São Paulo, v. 29, n. 2, p. 217-228, 2006. 
BONNET, A.; CURCIO, G. R.; LAVORANTI, O. J.; GALVÃO, F. Flora epifítica vascular em três unidades vegetacionais do Rio Tibagi, Paraná, Brasil. Rodriguésia, Rio de Janeiro, v. 62, n. 3, p. 491-498, 2011.

BORGO, M.; SILVA, S. M. Epífitos vasculares em fragmentos de Floresta Ombrófila Mista, Curitiba, Paraná, Brasil. Revista Brasileira de Botânica, São Paulo, v. 26, p. 391-401, 2003.

BREIER, T. B. Florística e ecologia de epífitos vasculares em uma Floresta Costeira do Sul do Brasil. 1999. 83 f. Dissertação (Mestrado) - Universidade Federal do Rio Grande do Sul, Porto Alegre. 1999.

BREIER, T. B. O epifitismo vascular nas Florestas do Sudoeste do Brasil, São Paulo, SP. 2005. 139 f. Tese (Doutorado em Biologia Vegetal) - Universidade Estadual de Campinas, Campinas. 2005.

BRUM-FIGUEIRÓ, A. C.; CITADINI-ZANETTE, V. Árvores de um fragmento florestal urbano no município de Criciúma, Santa Catarina. Revista Brasileira de Plantas Medicinais, São Paulo, v. 10, p. 56-67, 2008.

CAGLIONI, E.; BONNET, A.; SCHMITT, J. L.; CRISTOFOLINI, C.; ANDRADE, S.; CADORIN, T. J.; OLIVEIRA, C. P. L; GROSCH, B.; GASPER, A. L.; UHLMANN, A.; SEVEGNANI, L.; VIBRANS, A. C. Epífitos vasculares predominantes em zonas ecológicas de forófitos, Santa Catarina, Brasil. Revista de Estudos Ambientais, Blumenau, v. 14, n. 1, p. 28-42, 2012.

CALLEGARI-JACQUES, S. M. Bioestatística: princípios e aplicações. Porto Alegre: Artmed, 2003. 256 p.

CATLING, P. M.; LEFKOVITCH, L. P. Associations of vascular epiphytes in a Guatemalan cloud forest. Biotropica, Washington, v. 21, p. 35-40, 1989.

CMC. Câmara Municipal de Criciúma. Lei Municipal no 2.856 . 2006. Disponível em: <http:camara.plugin.com.br/mostra_lei. asp?lei=2856> . Acesso em: 12 out. 2009.

COIMBRA FILHO, A. F.; ALDRIGHI, A. D. Restabelecimento da fauna do Parque Nacional da Tijuca. Brasil Florestal, São Paulo, v. 3, n. 11, p. 19-33, 1972.

COTTAM, G.; CURTIS, J. T. The use of distance measurements in phytosociological sampling. Ecology, New York, v. 37, n. 37, p. 451-460, 1956.

COXSON, D. S.; NADKARNI, N. M. Ecological roles of epiphytes in nutrient cycles of forest ecossystem. In: LOWMAN, M. D.; NADKARNI, N. M. (Ed.). Forest canopies. San Diego: Academic Press, 1995. p. 495-593.

DETTKE, G. A.; ORFRINI, A. C.; MILANEZE-GUTIERRE, M. A. Composição florística e distribuição de epífitas vasculares em um remanescente alterado de Floresta Estacional Semidecidual no Paraná, Brasil. Rodriguésia, Rio de Janeiro, v. 59, n. 4, p. 859-872, 2008.

DIAS, A. S. Ecologia de epífitas vasculares em uma área de Mata Atlântica do Parque Nacional da Serra dos Órgãos, Teresópolis, RJ. 2009. 81 f. Dissertação (Mestrado em Ciências Ambientais e Florestais) - Universidade Federal Rural do Rio de Janeiro, Seropédia. 2009.

DISLICH, R.; MANTOVANI, W. Flora de epífitas vasculares da Reserva da Cidade Universitária "Armando de Oliveira Salles" (São Paulo, Brasil). Boletim de Botânica da Universidade de São Paulo, São Paulo, v. 17, p. 61-83, 1998.
DITTRICH, V. A. O.; KOZERA, C.; SILVA, S. M. Levantamento florístico de epífitos vasculares no Parque Barigüi, Paraná, Brasil. Iheringia, Série Botânica, Porto Alegre, v. 52, p. 11-22, 1999.

EPAGRI. Empresa de Pesquisas Agropecuária e de Extensão Rural de Santa Catarina. Secretaria de Estado do Desenvolvimento Rural e da Agricultura. Dados e informações bibliográficas da Unidade de Planejamento Regional Litoral Sul Catarinense - UPR 8. Florianópolis: EPAGRI, 2001. 77 p.

FILGUEIRAS, T. S.; NOGUEIRA, P. E.; BROCHADO, A. L.; GUALA II, G. F. Caminhamento: um método expedito para levantamentos florísticos qualitativos. Caderno de Geociências, Rio de Janeiro, n. 12, p. 39-43, 1994.

FISCHER, E. A.; ARAÚJO, A. C. Spatial organization of a bromeliad community in the Atlantic Rainforest, southeastern Brazil. Journal of Tropical Ecology, Cambridge, v. 11, p. 550567, 1995.

FONTOURA, T.; SYLVESTRE, L. S.; VAZ, A. M. S.; VIEIRA, C. M. Epífitas vasculares, hemiepífitas e hemiparasitas da Reserva Ecológica de Macaé de Cima. In: LIMA, H. C.; GUEDES-BRUNI, R. R. (Ed.). Serra de Macaé de Cima: diversidade florística e conservação em Mata Atlântica. Rio de Janeiro: Editora do Jardim Botânico, 1997. p. 89-101.

FREIBERG, M. Spatial distribution of vascular epiphytes on three emergent canopy trees in French Guiana. Biotropica, Washington, v. 28, n. 3, p. 345-355, 1996.

GARCIA-FRANCO, J. G.; RICO-GRAY, V. Experiments on seed dispersal and deposition patterns of epiphytes: the case of Tillandsia deppeana Steudel (Bromeliaceae). Phytologia, Beltsville, v. 65, p. 73-78, 1988.

GENTRY, A. H.; DODSON, C. H. Diversity and biogeography of neotropical vascular epiphytes. Annals of the Missouri Botanical Garden, Saint Louis, v. 74, p. 205-233, 1987.

GERALDINO, H. C. L.; CAXAMBÚ, M. G.; SOUZA, D. C. Composição florística e estrutura da comunidade de epífitas vasculares em uma área de ecótono em Campo Mourão, PR, Brasil. Acta Botanica Brasilica, São Paulo, v. 24, n. 2, p. 469-482, 2010. GIONGO, C.; WAECHTER, J. L. Composição florística e estrutura comunitária de epífitos vasculares em uma floresta de galeria na Depressão Central do Rio Grande do Sul. Revista Brasileira de Botânica, São Paulo, v. 27, n. 3, p. 563-572, 2004.

GONÇALVES, C. N.; WAECHTER, J. L. Aspectos florísticos e ecológicos de epífitos vasculares sobre figueiras isoladas no norte da planície costeira, RS. Acta Botanica Brasilica, São Paulo, v. 17, n. 1, p. 429-441, 2003.

HAMMER, O.; HARPER, D. A. T.; RYAN, P. D. PAST: Paleontological Statistics. Versão 1.89, 2009. Disponível em: $<$ http://folk.uio.no/ohammer/past>. Acesso em: 10 jul. 2011.

HEFLER, S. M.; FAUSTIONI, P. Levantamento florístico de epífitos vasculares do Bosque São Cristóvão, Curitiba, Paraná, Brasil. Revista Estudos de Biologia, Curitiba, v. 26, n. 54, p. 11-19, 2004.

HERWITZ, S. R.; SLYE, R. E. Spatial variability in the interception of inclined rainfall by a tropical rainforest canopy. Selbyana, Sarasota, v. 13, p. 62-71, 1992.

HIETZ, P.; HIETZ-SEIFERT, U. Composition and ecology of vascular epiphyte communities along an altitudinal gradient in central Veracruz, México. Journal of Vegetation Science, Xalapa, v. 6, p. 487-498, 1995. 
HIETZ, P. Population dynamics of epiphytes in a Mexican humid montane forest. Journal of Ecology, Oxford, v. 85, n. 6, p. 767-775, 1997.

HOELTGEBAUM, M. P. Composição florística e distribuição espacial de bromélias epifíticas em diferentes estádios sucessionais da floresta ombrófila densa, Parque Botânico do Morro Baú, Ilhota/SC. 2003. 138 f. Dissertação (Mestrado em Biologia Vegetal) - Universidade Federal de Santa Catarina, Florianópolis. 2003.

INGRAM, S. W.; NADKARNI, N. M. Composition and distribution of epiphytic organic matter in a neotropical cloud forest, Costa Rica. Biotropica, Washington, v. 25, p. 370-383, 1993.

JOHANSSON, D. R. Ecology of vascular epiphytes in West African rain forest. Acta Phytogeographica Suecica, Uppsala, v. 59, p. 1-136, 1974.

KERSTEN, R. A. Epifitismo vascular na bacia do Alto Iguaçu, Paraná. 2006. 231 f. Tese (Doutorado em Engenharia Florestal) Universidade Federal do Paraná, Curitiba. 2006.

KERSTEN, R. A.; KUNIYOSHI, Y. S. Conservação das florestas na bacia do alto Iguaçu, Paraná: avaliação da comunidade de epífitas vasculares em diferentes estágios serais. Floresta, Curitiba, v. 39, p. 51-66, 2009.

KERSTEN, R. A.; SILVA, S. M. Composição florística e estrutura do componente epifítico vascular em floresta da planície litorânea na Ilha do Mel, Paraná, Brasil. Revista Brasileira de Botânica, São Paulo, v. 24, n. 2, p. 213-226, 2001.

KERSTEN, R. A.; SILVA, S. M. Florística e estrutura do componente epifítico vascular em Floresta Ombrófila Mista Aluvial do rio Birigui, Paraná, Brasil. Revista Brasileira de Botânica, São Paulo, v. 25, n. 3, p. 259-267, 2002

KERSTEN, R. A.; WAECHTER, H. L. Florística e estrutura de epífitos vasculares na transição entre as florestas ombrófilas densa e mista da vertente da Serra do Mar paranaense, Brasil. In: FELFILI, J. M.; EISENLOHR, P. V.; MELO, M. M. R. F.; ANDRADE, L. A.; MEIRA NETO, J. A. A. (Ed.). Fitossociologia no Brasil: métodos e estudos de casos. Viçosa: Ed. UFV, 2011. p. 479-503.

KOTTEK, M. World map of the Köppen-Geiger climate classification updated. Meteorologische Zeitschrift, Viena, v. 15, n. 3, p. 259-263, 2006.

KRESS, W. J. The systematic distribution of vascular epiphytes: an update. Selbyana, Sarasota, v. 9, p. 2-22, 1986.

MADISON, M. Vascular epiphytes: the systematic occurrence and salient features. Selbyana, Sarasota, v. 2, p. 1-13, 1977.

MAGURRAN, A. F. Ecological diversity and its measurement. Princeton: Princeton Univesity Press, 1988. 179 p.

MUSSKOPF, E. L. Composição florística e distribuição ecológica de epífitos vasculares no Parque Estadual de Itapuã, Viamão, Rio Grande do Sul. 2006. 60 f. Dissertação (Mestrado em Botânica) Universidade Federal do Rio Grande do Sul, Porto Alegre. 2006.

NIEDER, J.; IBISCH, P. L.; BARTHLOTT, W. Biodiversidad de epífitas: una cuestión de escala. Revista del Jardim Botánico Nacional, La Habana, v. 18, p. 12-13, 1996.

NIEDER, J.; PROSPERÍ, J.; MICHALOUD, G. Epiphytes and their contribution to canopy diversity. Plant Ecology, Dordrecht, v. 153, p. 51-63, 2001.

PETEAN, M. P. O componente epifítico vascular em Floresta Ombrófila Densa no litoral paranaense: análise florística, estrutural e de biomassa. 2009. 75 f. Tese (Doutorado em Engenharia Florestal) - Universidade Federal do Paraná, Curitiba. 2009.
PIELOU, E. C. Ecological diversity. New York: Wiley Interscience, $1975.165 \mathrm{p}$.

REITZ, R. Bromeliáceas e a malária: bromélia endêmica. Flora Ilustrada Catarinense, Itajaí, v. 1, p. 1-518, 1983.

ROCHA, E. Estrutura da sinúsia herbácea terrícola do Parque Ecológico Municipal José Milanese, Criciúma, Santa Catarina. 2007. 32 f. Monografia (Graduação em Ciências Biológicas) Universidade do Extremo Sul Catarinense, Criciúma. 2007.

ROGALSKI, J. M.; ZANIN, E. M. Composição florística de epífitos vasculares no estreito de Augusto César, Floresta Estacional Decidual do Alto Uruguai, RS, Brasil. Revista Brasileira de Botânica, São Paulo, v. 26, n. 4, p. 551-556, 2003.

RUDOLPH, D.; RAUER, G.; NIEDER, J.; BARTHLOTT, W. Distributional patterns of epiphytes in the canopy and phorophyte characteristics in a western Andean rain forest in Ecuador. Selbyana, Sarasota, v. 19, n. 1, p. 27-33, 1998.

SANTOS, A. C. L. Composição florística e estrutura da comunidade de epífitas vasculares associadas a trilhas no Parque Estadual das Fontes do Ipiranga, São Paulo, SP, Brasil. 2008. 72 f. Dissertação (Mestrado em Biodiversidade Vegetal e Meio Ambiente) - Instituto de Botânica da Secretaria do Meio Ambiente, São Paulo. 2008.

SANTOS, C. G. M. Distribuição espacial, fenologia e polinização de Bromeliaceae na Mata Atlântica do Alto da Serra de Paranapiacaba, SP. 2000. 112 f. Tese (Doutorado em Biologia Vegetal) - Universidade Estadual de Campinas, Campinas. 2000.

SHAW, D. C. Vertical organization of canopy biota. In: LOWMAN, M. D.; REINKER, H. B. Forest Canopies. London: Elsevier Academic, 2004. p. 73-101.

SILVA, R. T. Florística e estrutura da sinúsia arbórea de um fragmento urbano de floresta ombrófila densa do município de Criciúma, Santa Catarina. 2006. 61 f. Dissertação (Mestrado em Ciências Ambientais) - Universidade do Extremo Sul Catarinense, Criciúma. 2006.

TER STEEGE, H.; CORNELISSEN, J. H. C. Distribution and ecology of vascular epiphytes in lowland rain forest of Guyana. Biotropica, Washington, v. 21, n. 4, p. 331-339, 1989.

VENTURA, G. M.; DIAS, J. M. M.; TEIXEIRA, L. S.; CARVALHOS, S. V.; MOTOIKE, Y. S.; NOVAIS, F. R; CECON, R. P. Organogênese in vitro a partir de gemas apicais e axilares de plantas adultas de orquídeas do grupo Cattleya. Revista Ceres, Viçosa, v. 47, p. 613-628, 2002.

WAECHTER, J. L. Epífitos vasculares da mata paludosa do Faxinal, Torres, Rio Grande do Sul, Brasil. Iheringia, Série Botânica, Porto Alegre, v. 34, p. 39-49, 1986.

WAECHTER, J. L. O epifitismo vascular na Planície Costeira do Rio Grande do Sul. 1992. 163 f. Tese (Doutorado em Ecologia e Recursos Naturais) - Universidade Federal de São Carlos, São Paulo. 1992.

WAECHTER, J. L. Epifitismo em uma floresta de restinga do Brasil subtropical. Ciência e Natura, Santa Maria, v. 20, p. 43-66, 1998.

YEATON, R. I.; GLADSTONE, D. E. The pattern of colonization of epiphytes on Calabash Trees (Crescentia alata HBK.) in Guanacaste Province, Costa Rica, Biotropica, Washington, v. 14, n. 2, p. $137-$ 140, 1982.

ZAR, J. H. Biostatistical analysis. 4. ed. New Jersey: Prentice Hall, 1999. $459 \mathrm{p}$. 